\title{
How to collect better data? Longer exposure times versus higher redundancy Michael Ruf ${ }^{1}$ \\ ${ }^{1}$ Bruker AXS Inc \\ michael.ruf@bruker.com
}

Solving and refining the three-dimensional atomic structure of a crystalline compound requires collecting accurate and precise diffraction data. However, every experiment that is based on intensity measurements will always be affected by a degree of uncertainty and no matter how careful the experiment is conducted, there is always error in a measurement. Error is not a "mistake" per se, it is part of the measuring process.

There are two broad classes of observational errors: random error and systematic error. Random error varies unpredictably from one measurement to another, while systematic error has the same value or proportion for every measurement.

Random error

The dominating source of random error in intensity measurements results from the quantum nature of X-rays. The error follows the Poisson distribution of counting statistics, which can be approximated by a Gaussian function. The value of the error is the square root of the number of photons and the relative random error of the measured intensity is

$1 /(\sqrt{ } \mathrm{n})$

where $\mathrm{n}$ is the number of photons. Random error not only results from fluctuations of a Bragg peak's intensity, but also from fluctuations of photons in the background beneath and around the peak. This means that to effectively measure small differences in diffraction intensities, large numbers of photons are required. To reach a $1 \%$ error for example at least 10,000 photons in a Bragg peak are required.

Systematic error. Systematic errors can be caused by simplified assumptions about the sample and diffraction geometry as well as diffraction physics, instrumentation, and from approximations during data processing. Examples are imprecise detector calibrations, variations in amplifier gain, crystal vibration, shutter error, non-uniform crystal rotation resulting from worn-out gears or unstable motor control. Improperly defined saturation limits, count-rate saturation or charge sharing. Other sources of systematic error are extinction during diffraction, as well as absorption. The consensus is that the effect of systematic error can be minimized by collecting redundant data by choosing several diffraction geometries recording multiple independent measurements of the same reflection. 